\title{
Comparative metabolomic analysis of HPAC cells following the acquisition of erlotinib resistance
}

\author{
SOOYEUN LEE ${ }^{1}$, WON-JUN JANG ${ }^{1}$, BOYEON CHOI ${ }^{1}$, SANG HOON JOO $^{2}$ and CHUL-HO JEONG ${ }^{1}$ \\ ${ }^{1}$ College of Pharmacy, Keimyung University, Dalseo, Daegu 42601; ${ }^{2}$ Department of Pharmacy, \\ Catholic University of Daegu, Gyeongsan-si, Gyeongbuk 38430, Republic of Korea
}

Received November 4, 2015; Accepted February 17, 2017

DOI: 10.3892/ol.2017.5940

\begin{abstract}
Pancreatic cancer is one of the most lethal types of cancer, due to difficulty in early detection and the limited efficacy of available treatments. Erlotinib is used to inhibit the epidermal growth factor receptor for the treatment of pancreatic cancer; however, erlotinib resistance is a major issue and the mechanisms underlying the development of erlotinib resistance remain unclear. To better understand the alterations in tumor metabolism by acquired resistance to erlotinib, an erlotinib-resistant pancreatic cancer cell line (HPAC-ER) was established, followed by a comparison of the metabolic characteristics between these cells and their erlotinib-sensitive parental cells (HPAC). This comparison was accomplished through mass spectrometry-based targeted metabolic profiling. Five metabolite groups (acylcarnitines, amino acids and biogenic amines, glycerophospholipids, sphingolipids and monosaccharides) were semi-quantified and compared statistically. These results revealed significant differences between the two groups of cells. A significant increase in the level of short-chain acylcarnitines and selected lysophosphatidylcholines, and a significant decrease in the level of acyl-alkyl-phosphatidylcholines and one sphingolipid, were observed in the HPAC-ER cells compared with the HPAC cells. The metabolic changes observed in the present study support the theory that there are increased metabolic demands in erlotinib-resistant cancer, reflecting the changes in acetyl-CoA-associated and choline phospholipid metabolism. These findings will aid in elucidating the changes that occur in pancreatic cancer metabolism through the acquired resistance
\end{abstract}

Correspondence to: Professor Sang Hoon Joo, Department of Pharmacy, Catholic University of Daegu, 13-13 Hayang-Ro, Hayang Eup, Gyeongsan-si, Gyeongbuk 38430, Republic of Korea

E-mail: sjoo@cu.ac.kr

Professor Chul-Ho Jeong, College of Pharmacy, Keimyung University, 1095 Dalgubeoldaero, Dalseo, Daegu 42601, Republic of Korea

E-mail: chjeong75@kmu.ac.kr

Key words: chemoresistance, erlotinib, pancreatic cancer, metabolomics, metabolite to erlotinib, and in the identification of biomarkers for the early detection of pancreatic cancer.

\section{Introduction}

Pancreatic cancer is one of the most lethal types of cancer, with the majority of patients suffering from inoperable disease at the time of diagnosis (1). Despite tremendous efforts in developing novel therapies for pancreatic cancer, particularly in the past two decades, the overall 5-year survival rate remains $<5 \%$ and the median survival period is 6 months following diagnosis (2). Given that one of the growth promoter/survival signaling pathways upregulated during pancreatic tumorigenesis is that of epidermal growth factor receptor (EGFR), targeting EGFR activity is considered to be a promising strategy for the treatment of pancreatic cancer, in addition to cytotoxic chemotherapy. To date, erlotinib, a small molecule tyrosine kinase inhibitor (TKI) for EGFR, in combination with gemcitabine chemotherapy, has been approved as a first-line therapy for locally advanced and metastatic pancreatic cancer. Although this regimen has prolonged the median survival period of patients with pancreatic cancer, the benefit is quite modest owing to the therapy resistance of this malignancy. Furthermore, only $25-30 \%$ of patients respond to this regimen and eventually exhibit cancer progression due to chemoresistance (3). In this regard, pancreatic cancer remains an intractable and recurrent metastatic cancer despite notable improvements in chemotherapeutic approaches. Therefore, there is a clear and distinct requirement to understand the underlying molecular mechanisms and biology of pancreatic cancer, including the genomic or metabolomic alterations responsible for chemoresistance, which is an essential issue to address in order to develop effective novel therapies for pancreatic cancer.

Metabolomics is an approach that enables the better understanding of the physiological or pathophysiological states of humans, animals or plants by identifying and/or quantifying known or novel metabolites in biological specimens, followed by statistical interpretation of changes in the metabolites in association with their biological systems. Over the last decade, publications in metabolomics for human urine, blood and cell culture samples have markedly increased $(4,5)$ in order to facilitate the understanding of human biology in health and disease. In particular, cell culture metabolomics serves a key 
role in enhancing the understanding of cell properties and functions depending on specific cellular phenotypes (5). Mass spectrometry (MS) is an important tool in metabolomics, because of the superior metabolite coverage made available by the technique due to improved sensitivity and reproducibility $(4,6)$. Thus, MS is the most appropriate approach for cell culture metabolomics, although its applications with cell lysates remain limited to in vitro measurement (5).

In the current study, erlotinib-resistant human pancreatic adenocarcinoma cells (HPAC-ER) were established in order to obtain the relevant metabolic signatures for the early detection of chemoresistance to erlotinib. To achieve this, the metabolic characteristics between erlotinib-sensitive (HPAC) and erlotinib-resistant (HPAC-ER) pancreatic cancer cells were compared by MS-based targeted metabolic profiling. The targeted metabolic analysis was performed with a commercial kit using a MS-based flow injection analysis (FIA) and an MS-based liquid chromatography (LC) to quantify the following five metabolite groups: Acylcarnitines; amino acids and biogenic amines, glycerophospholipids; sphingolipids; and monosaccharides. Throughout the use of this metabolomic approach, the deregulation of metabolic signaling pathways induced by the acquisition of resistance to erlotinib in pancreatic cancer was investigated.

\section{Materials and methods}

Materials. Erlotinib was purchased from LC Laboratories (Woburn, MA, USA). Halt ${ }^{\mathrm{TM}}$ Protease/Phosphatase Inhibitors Cocktail (100X), EDTA (100X) and the BCA protein assay kit were purchased from Thermo Fisher Scientific, Inc. (Waltham, MA, USA). MTT was purchased from Sigma-Aldrich (Merck KGaA, Darmstadt, Germany). The AbsoluteIDQ ${ }^{\circledR}$ p180 kit was obtained from Biocrates Life Sciences AG (Innsbruck, Austria). All solvents used for MS were of high-performance liquid chromatography grade.

Cell culture. The human pancreatic adenocarcinoma cell line HPAC was obtained from the American Type Culture Collection (Manassas, VA, USA) and cultured in RPMI-1640 medium with L-glutamine supplemented with $10 \%$ FBS (Gibco; Thermo Fisher Scientific, Inc.) and 1\% penicillin/streptomycin (Hyclone; GE Healthcare Life Sciences, Logan, UT, USA). Erlotinib-resistant HPAC cells (HPAC-ER) were generated through continuous exposure of parental HPAC cells to erlotinib for $>6$ months. Starting with an erlotinib concentration of $0.1 \mu \mathrm{M}$, the exposure dose was doubled every 2 weeks until a final concentration of $10 \mu \mathrm{M}$ was achieved. HPAC-ER cells were cultured in the same medium, with the addition of $1 \mu \mathrm{M}$ erlotinib. All cells were cultured as monolayers at $37^{\circ} \mathrm{C}$ in a humidifier incubator with $5 \% \mathrm{CO}_{2}$.

Cell viability assay. Cell viability was measured using the MTT assay. HPAC or HPAC-ER cells $\left(1 \times 10^{3}\right.$ cells/well) were treated with $0.1-10 \mu \mathrm{M}$ of erlotinib and incubated for $72 \mathrm{~h}$ at $37^{\circ} \mathrm{C}$. Following this, the media was replaced with the fresh RPMI-1640 medium supplemented MTT (0.5 mg/ml MTT; $100 \mu \mathrm{l} /$ well) and incubated for $4 \mathrm{~h}$ at $37^{\circ} \mathrm{C}$. The medium was subsequently aspirated from the wells, $100 \mu \mathrm{l}$ dimethyl sulfoxide (DMSO) added and the plates agitated for $3 \mathrm{~min}$. The absorbance at $565 \mathrm{~nm}$ was then read using a Tecan Infinite ${ }^{\circledR}$ F200 PRO plate reader (Promega Corporation, Madison, WI, USA). Results are presented as the percentage of absorbance relative to cells incubated with DMSO alone.

Soft agar colony formation assay. HPAC or HPAC-ER cells $\left(8 \times 10^{3}\right.$ cells/well) were suspended in Basal Medium Eagle (BME; $1 \mathrm{ml}$ with $10 \%$ FBS and $0.33 \%$ bacto agar) and plated over a layer of solidified agar (BME with 10\% FBS and $0.5 \%$ bacto agar). The cultures were maintained at $37^{\circ} \mathrm{C}$ in an incubator with $5 \% \mathrm{CO}_{2}$ for 7 days, and the colonies were observed using a light microscope (magnification, $\mathrm{x} 40$ ).

Metabolomic analysis. For the determination of intracellular metabolites, cell culture lysates were prepared using a modified extraction protocol, as described previously (7). Following removal of the media, the HPAC and HPAC-ER cells were washed two times with ice-cold PBS and lyzed in $10 \mathrm{mM}$ phosphate buffer (Sigma-Aldrich; Merck KGaA). Then, three cycles of sonication $\left(40 \mathrm{kHz} ; 25^{\circ} \mathrm{C} ; 15 \mathrm{sec}\right)$ followed by a freeze-thaw cycle (liquid nitrogen for $30 \mathrm{sec}$ followed by instantly thawing in a $98^{\circ} \mathrm{C}$ heat block) were performed, and the samples were centrifuged to collect the cell lysates $\left(20,000 \times \mathrm{g}, 10 \mathrm{~min}, 4^{\circ} \mathrm{C}\right)$.

The metabolomic analysis in the prepared cell lysates was performed using the AbsoluteIDQ p180 kit, which allowed the simultaneous quantification of a total of 186 metabolites (40 acylcarnitines, 41 amino acids and biogenic amines, 90 glycerophospholipids and 15 sphingolipids) and a sum of hexoses (including glucose), including glucose (Table I). The kit was used with a 4000 QTRAP ${ }^{\circledR}$ Mass Spectrometer (AB Sciex, Framingham, MA, USA) in multiple reaction monitoring detection mode with electrospray ionization (ESI) at Inha University Hospital Clinical Trial Center (Incheon, Korea). Amino acids and biogenic amines were injected into the mass spectrometer using FIA and the other groups of metabolites were injected via LC. The kit was validated using MetVal ${ }^{\mathrm{TM}}$ software (Biocrates Life Sciences AG) and the analytical results were processed using Analyst ${ }^{\mathrm{TM}}$ (version 1.6.2; AB Sciex) and $\mathrm{MetVal}^{\mathrm{TM}}$ software (Biocrates Life Sciences AG). The quantitative metabolite results were normalized to total protein concentrations of cell lysates, which were determined using the BCA Protein assay kit.

Statistical analysis. Statistical analysis of the quantitative metabolite results was performed using Mass Profiler Professional software (version B.12.6.1; Agilent Technologies, Inc., Santa Clara, CA, USA). The comparison of metabolites between the HPAC and HPAC-ER cells was performed using multivariate analysis with principal component analysis (PCA) and partial least squares discriminant analysis (PLS-DA). A Student's t-test or one-way analysis of variance with a multiple testing correction (Benjamini-Hochberg false discovery rate) were used to determine significant differences in the quantitative results of the metabolites, with a $\log _{2}$ fold-change $>1$ and $\mathrm{P}$-value $\leq 0.001$ considered to indicate a statistically significant difference. Based on these criteria, a volcano plot was generated. A heat map was also produced by the unsupervised hierarchical clustering of metabolite signatures. 
Table I. Number of metabolites investigated in HPAC and HPAC-ER cells.

\begin{tabular}{lccc}
\hline Metabolite group & $\begin{array}{c}\text { Total number of } \\
\text { metabolites }\end{array}$ & $\begin{array}{c}\text { Number of semi- } \\
\text { quantified metabolites }\end{array}$ & $\begin{array}{c}\text { Number of significantly } \\
\text { changed metabolites } \\
\text { (HPAC vs. HPAC-ER cells) }\end{array}$ \\
\hline Acylcarnitines & 40 & 5 & 2 \\
Amino acids and biogenic amines & 41 & 33 & 5 \\
Glycerophospholipids & 90 & 84 & 37 \\
Sphingolipids & 15 & 14 & 1 \\
Monosaccharides & 1 & 137 & 0 \\
Total & 187 & 45 \\
\hline
\end{tabular}

HPAC, human pancreatic adenocarcinoma cells; HPAC-ER, HPAC-erlotinib resistant cells.

\section{Results and discussion}

Successful establishment of HPAC-ER cells. To establish the HPAC-ER cell line, HPAC cells were cultured using a stepwise selection method, with media containing an increasing erlotinib concentration (0.01-10 $\mu \mathrm{M})$. The resultant HPAC-ER cells were characterized by distinct morphological changes, including a loss of cell-cell adhesion and scattered pattern of distribution compared with their parental cell line, HPAC (Fig. 1A). The quantification of cell viability at different concentrations of erlotinib also verified the successful generation of erlotinib-resistance (Fig. 1B). The viability of HPAC cells gradually decreased to $<50 \%$ following treatment with 1-5 $\mu \mathrm{M}$ range of erlotinib, whereas the viability of HPAC-ER cells remained relatively unchanged; the HPAC-ER cells were significantly more resistant to erlotinib $(>1 \mu \mathrm{M})$ compared with their parental cells.

HPAC-ER cells have increased colony-forming ability. The ability of transformed cells to form colonies in soft agar is associated with in vivo tumorigenesis and is frequently used as a surrogate in vitro assay for an in vivo phenotype (7). Therefore, an anchorage-independent colony formation assay in soft agar was performed (Fig. 1C). This revealed that HPAC-ER cells formed a markedly higher number of colonies compared with HPAC cells, suggesting that the molecular alterations responsible for the tumorigenic phenotype occur during the acquisition of resistance to erlotinib in HPAC-ER cells.

\section{Metabolomic analysis}

Semi-quantification of metabolite concentrations. To understand the changes in tumor metabolism, and investigate the metabolic differences between HPAC and HPAC-ER cells in relation to their sensitivity to erlotinib, MS-based targeted metabolomic profiling analysis was performed. A total of 137 metabolites were semi-quantified (Table I). In MS using ESI, the matrix effect is a major issue that affects accurate quantification (8). Even though quantitative results of concentrations of metabolites were obtained from the cell lysates, these were not considered to be an accurate indication of the concentration of metabolites in the cells because the calibrators (standards only) used for quantification were not matrix-matched. Therefore, $\log _{2}$ fold-change values were used to describe the concentrations of significantly altered metabolites between the HPAC and HPAC-ER cells (Table II).

Metabolite concentrations were significantly different between HPAC and HPAC-ER cells. From the statistical analysis, the concentrations of 45 metabolites (2 acylcarnitines, 5 amino acids and biogenic amines, 37 glycerophospholipids and 1 sphingolipid) were significantly different between the HPAC and HPAC-ER cells (Tables I and II). The semi-quantitative results of these metabolites revealed a marked differentiation between HPAC and HPAC-ER cells, as presented in the PCA score plot (Fig. 2A). This differentiation was primarily represented by the first principal component (PC1), which was $96.38 \%$ of the observed variations. A supervised multivariate analysis, PLS-DA, also revealed a distinct separation between the two groups of the cells $\left(\mathrm{R}^{2} 0.994 ; \mathrm{Q}^{2}=0.993\right.$; Fig. $\left.2 \mathrm{~B}\right)$. The volcano plot displays the production signatures of significantly increased or decreased metabolites in the HPAC-ER compared with the HPAC cells (Fig. 3A). A total of 12/45 (27\%) and 33/45 (73\%) of the significantly differentially produced metabolites were increased and decreased, respectively. The graphic heat map produced depicts the levels of the significantly differentially produced metabolites in each sample of the two groups, in addition to the variation in the amount of each metabolite between samples (Fig. 3B).

Table II presents the list of metabolites that were significantly differently produced between HPAC and HPAC-ER cells. Two short-chain acylcarnitines, acetylcarnitine (C2) and propionylcarnitine (C3), were present in the HPAC-ER cells at significantly higher levels compared with the HPAC cells (Table II), while the other longer-chain acylcarnitines were below the limit of quantification, excluding hexadecanolycarnitine (C16), which was not significantly different (data not shown). The level of carnitine (C0) was not significantly different between the two cell lines (data not shown). Acylcarnitines are essential compounds for mitochondrial fatty acid oxidation. Long-chain acylcarnitines are used for the formation of ATP, the energy currency in cells. Acetyl coenzyme A (acetyl-CoA), produced during the $\beta$-oxidation of fatty acids in the mitochondria, is converted to acetylcarnitine by carnitine acetyl transferase. In addition, acetylcarnitine from peroxisomes is exported to the mitochondria for the 
A

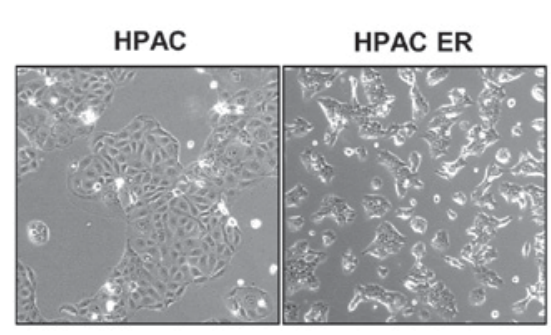

B

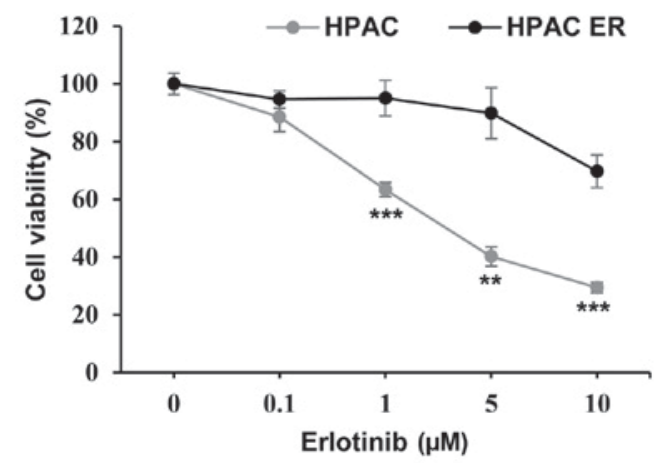

C

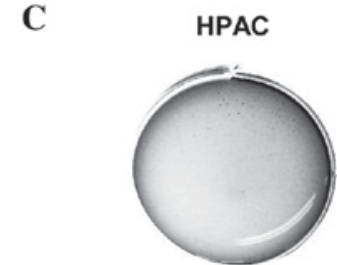

HPAC ER

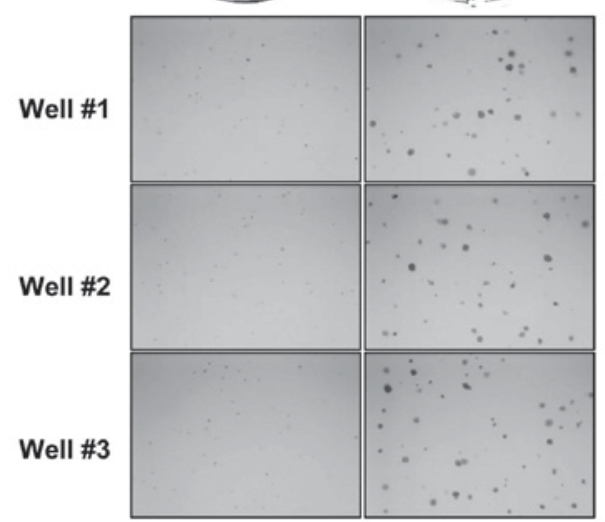

Figure 1. Establishment and characterization of HPAC-ER cells. HPAC and HPAC-ER cell (A) Morphology (magnification, x100) and (B) cell viability following erlotinib treatment. Data is presented as the mean \pm standard deviation of three independent experiments. ${ }^{* *} \mathrm{P}<0.01,{ }^{* * *} \mathrm{P}<0.001$ vs. the HPAC cell group (Student's t-test). (C) Soft agar colony formation assay (magnification, $\mathrm{x} 40$ ). HPAC-ER, erlotinib-resistant human pancreatic adenocarcinoma cells.

metabolism of long-chain acylcarnitines $(9,10)$. Therefore, the increased abundance of acetylcarnitine in HPAC-ER cells indicates that they have an elevated metabolic capacity and produce more energy compared with their erlotinib-sensitive counterparts.

Deregulation of metabolite production contributes to the chemoresistance of cancer cells. Deregulation of amino acids and biogenic amines may be associated with the drug resistance of cancer cells (11-13). The abundance of glutamate from the conversion of glutamine to glutamate is a well-known characteristic of cancer cells that possess high glutaminase activity and low glutamine synthase activity (11). Consistent with this, a previous study indicated that glutamate levels were significantly higher in erlotinib-resistant non-small cell lung cancer cells (PC-9ER) compared with their erlotinib-sensitive parental cell line (PC-9) (14). In addition, a recent study reported decreased glutamine levels in two different gemcitabine-resistant human pancreatic cancer cell lines (SUIT-2-GR and CAPAN-1-GR) compared with their gemcitabine-sensitive parental cell lines (15). Glutamate serves a role in a variety of metabolic signaling pathways, including those for taurine, hypotaurine, D-glutamine, D-glutamate and glutathione metabolism. In the present study, glutamate and taurine were upregulated in the HPAC-ER compared with the HAPC cells (Table II). Since taurine is a fundamental amino acid for cell development, nutrition and survival (16), the increased levels of taurine and glutamate may contribute to the tumorigenic characteristics of HPAC-ER cells.

$\alpha$-Aminoadipic acid, which mediates the synthesis of lysine and acetyl-CoA, was previously identified as one of the biomarkers of cancer from metabolic footprinting in Kruppel like factor 4-deficient mouse embryonic fibroblasts (17). Similarly, the data from the present study demonstrated that $\alpha$-aminoadipic acid was present at a significantly higher level in the HPAC-ER cells compared with the HPAC cells (Table II).

According to a previous review of the role of two monoamine neurotransmitters, serotonin and dopamine, in regulation of tumor behavior, there have been reports of metabolites serving opposing roles in cancer; serotonin has been revealed to stimulate while dopamine has been demonstrated to inhibit tumor growth (18). Notably, the present study demonstrated that the level of serotonin was significantly 
Table II. Significantly changed metabolites in HPAC and HPAC-ER cells.

\begin{tabular}{|c|c|c|c|}
\hline Metabolite & P-value & $\log 2$ fold-change & Production change \\
\hline Acetylcarnitine & $9.86 \mathrm{E}-07$ & 1.24 & Up \\
\hline Propionylcarnitine & $3.18 \mathrm{E}-10$ & 2.93 & Up \\
\hline Glutamate & $1.75 \mathrm{E}-07$ & 1.04 & Up \\
\hline Asymmetric dimethylarginine & $2.79 \mathrm{E}-11$ & ND & Up \\
\hline$\alpha$-Aminoadipic acid & $2.47 \mathrm{E}-05$ & ND & Up \\
\hline Serotonin & $2.60 \mathrm{E}-14$ & ND & Down \\
\hline Taurine & $1.27 \mathrm{E}-10$ & 1.79 & Up \\
\hline LysoPC a C20:4 & $3.91 \mathrm{E}-06$ & 1.66 & Up \\
\hline LysoPC a C26:0 & $1.53 \mathrm{E}-06$ & 1.25 & Up \\
\hline LysoPC a C26:1 & $2.01 \mathrm{E}-07$ & 1.39 & Up \\
\hline $\mathrm{PC}$ aa $\mathrm{C} 30: 2$ & $2.16 \mathrm{E}-04$ & 1.20 & Up \\
\hline $\mathrm{PC}$ aa $\mathrm{C} 32: 3$ & $6.04 \mathrm{E}-09$ & 1.06 & Up \\
\hline $\mathrm{PC}$ aa C34:1 & $4.33 \mathrm{E}-11$ & -1.16 & Down \\
\hline $\mathrm{PC}$ aa $\mathrm{C} 36: 2$ & $1.06 \mathrm{E}-12$ & -1.71 & Down \\
\hline $\mathrm{PC}$ aa $\mathrm{C} 36: 5$ & $8.63 \mathrm{E}-12$ & 1.43 & Up \\
\hline PC aa C38:0 & $1.29 \mathrm{E}-09$ & -1.13 & Down \\
\hline $\mathrm{PC}$ ae $\mathrm{C} 30: 0$ & $1.03 \mathrm{E}-14$ & -2.40 & Down \\
\hline PC ae C30:1 & $2.62 \mathrm{E}-10$ & -1.06 & Down \\
\hline $\mathrm{PC}$ ae $\mathrm{C} 32: 1$ & $3.53 \mathrm{E}-14$ & -2.46 & Down \\
\hline $\mathrm{PC}$ ae $\mathrm{C} 32: 2$ & $1.74 \mathrm{E}-10$ & -1.09 & Down \\
\hline $\mathrm{PC}$ ae $\mathrm{C} 34: 0$ & $1.34 \mathrm{E}-10$ & -1.21 & Down \\
\hline $\mathrm{PC}$ ae $\mathrm{C} 34: 1$ & $2.79 \mathrm{E}-13$ & -2.24 & Down \\
\hline PC ae C34:2 & $5.19 \mathrm{E}-13$ & -1.93 & Down \\
\hline PC ae C34:3 & $6.20 \mathrm{E}-10$ & -1.08 & Down \\
\hline PC ae C36:1 & $4.51 \mathrm{E}-12$ & -1.81 & Down \\
\hline $\mathrm{PC}$ ae $\mathrm{C} 36: 2$ & $3.41 \mathrm{E}-13$ & -1.89 & Down \\
\hline $\mathrm{PC}$ ae $\mathrm{C} 36: 3$ & $1.02 \mathrm{E}-12$ & -2.02 & Down \\
\hline $\mathrm{PC}$ ae $\mathrm{C} 36: 4$ & $1.64 \mathrm{E}-11$ & -1.68 & Down \\
\hline PC ae C38:1 & $2.93 \mathrm{E}-10$ & -1.24 & Down \\
\hline PC ae C38:2 & $2.55 \mathrm{E}-11$ & -1.39 & Down \\
\hline $\mathrm{PC}$ ae $\mathrm{C} 38: 3$ & $1.70 \mathrm{E}-11$ & -1.18 & Down \\
\hline PC ae C38:4 & $1.84 \mathrm{E}-11$ & -1.62 & Down \\
\hline $\mathrm{PC}$ ae $\mathrm{C} 38: 5$ & $2.24 \mathrm{E}-10$ & -1.32 & Down \\
\hline PC ae C40:1 & $2.51 \mathrm{E}-10$ & -1.18 & Down \\
\hline PC ae C40:2 & $1.39 \mathrm{E}-12$ & -1.98 & Down \\
\hline PC ae C40:3 & $3.55 \mathrm{E}-10$ & -1.06 & Down \\
\hline PC ae C40:6 & $5.69 \mathrm{E}-11$ & -1.36 & Down \\
\hline $\mathrm{PC}$ ae $\mathrm{C} 42: 1$ & $4.98 \mathrm{E}-13$ & -1.78 & Down \\
\hline $\mathrm{PC}$ ae $\mathrm{C} 42: 2$ & $1.10 \mathrm{E}-13$ & -2.93 & Down \\
\hline $\mathrm{PC}$ ae $\mathrm{C} 42: 3$ & $3.42 \mathrm{E}-11$ & -1.86 & Down \\
\hline PC ae C44:3 & $7.23 \mathrm{E}-13$ & -2.15 & Down \\
\hline $\mathrm{PC}$ ae $\mathrm{C} 44: 4$ & $7.06 \mathrm{E}-11$ & -1.86 & Down \\
\hline $\mathrm{PC}$ ae $\mathrm{C} 44: 5$ & $7.16 \mathrm{E}-12$ & -1.78 & Down \\
\hline $\mathrm{PC}$ ae $\mathrm{C} 44: 6$ & $2.01 \mathrm{E}-08$ & -1.22 & Down \\
\hline SM C24:0 & $3.22 \mathrm{E}-11$ & -1.43 & Down \\
\hline
\end{tabular}

Phosphatidylcholine, PC; SM, sphingomyeline; aa, diacyl; ae, acyl-alkyl; ND, not detected.

decreased in the HPAC-ER cells compared with the HPAC cells (Table II). Few studies investigating the role served by serotonin in chemoresistance have been reported; thus, the biological significance of the decreased level of serotonin observed in HPAC-ER cells remains unclear without further investigation. 
A
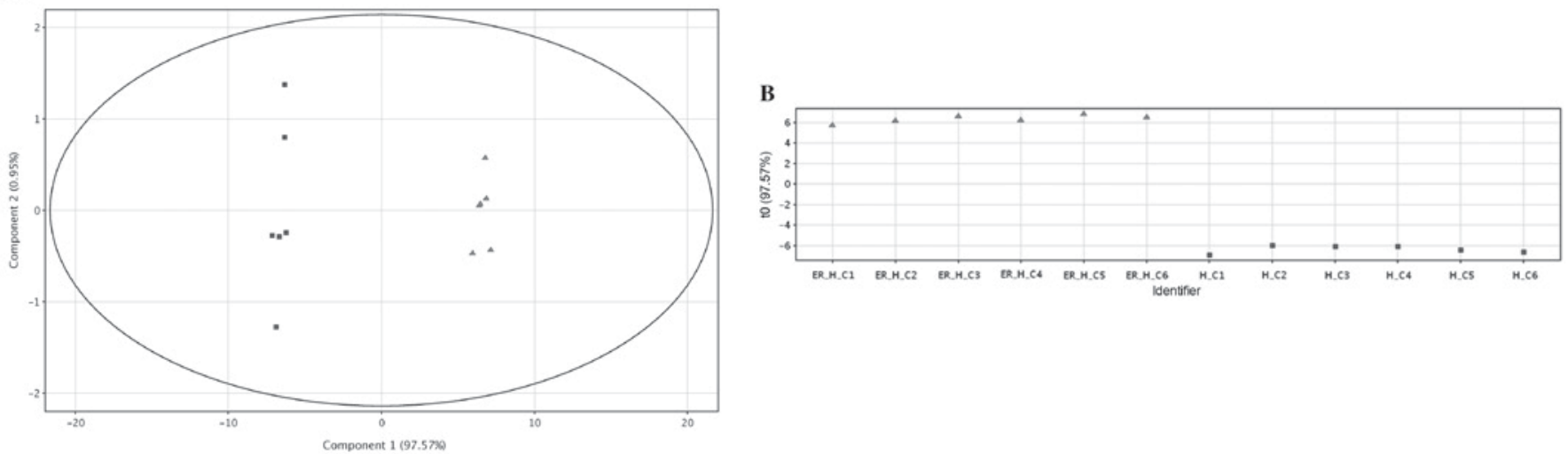

Figure 2. Score plots for significantly differentially produced metabolites between human pancreatic adenocarcinoma (HPAC; squares) and erlotinib-resistant HPAC (triangles) cells. (A) Principal component analysis and (B) partial least squares discriminant analysis.

A

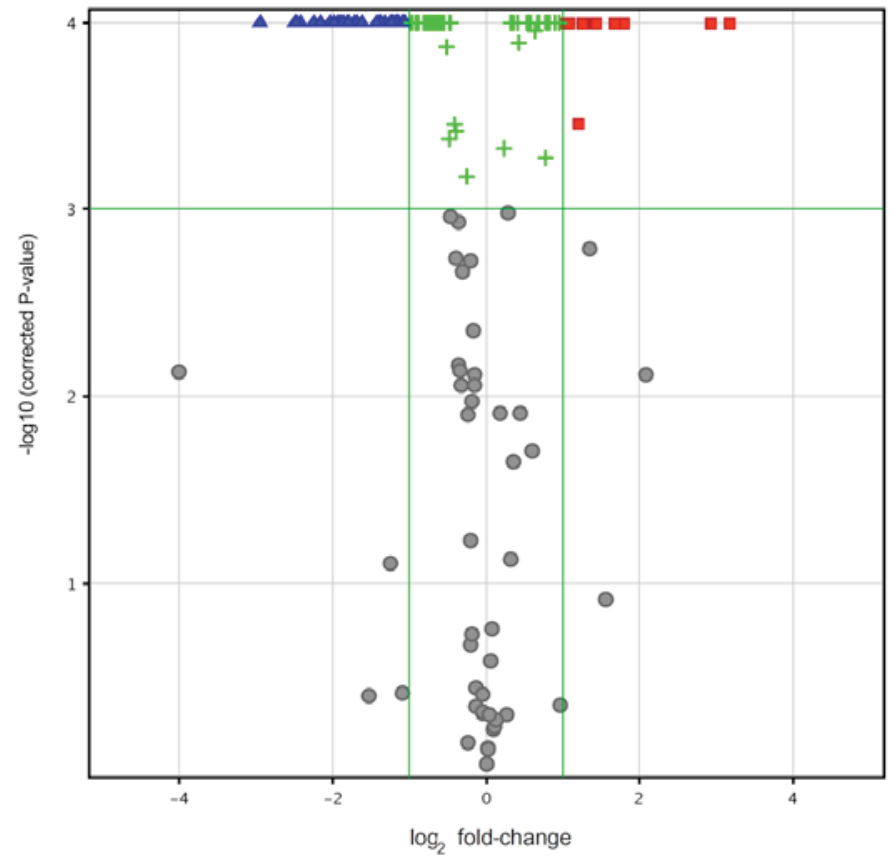

4: downregulated metabolites; passed cut-offs $\left(\log _{2}\right.$ fold-change $>2$ and $\left.p<0.001\right)$

": upregulated metabolites; passed cut-offs ( $\log _{2}$ fold-change $>2$ and $\left.p<0.001\right)$

- not changed metabolites; failed to pass p-value cut-off;

$+:$ not changed metabolites; passed p-value cut-off but failed to pass fold change cut-off

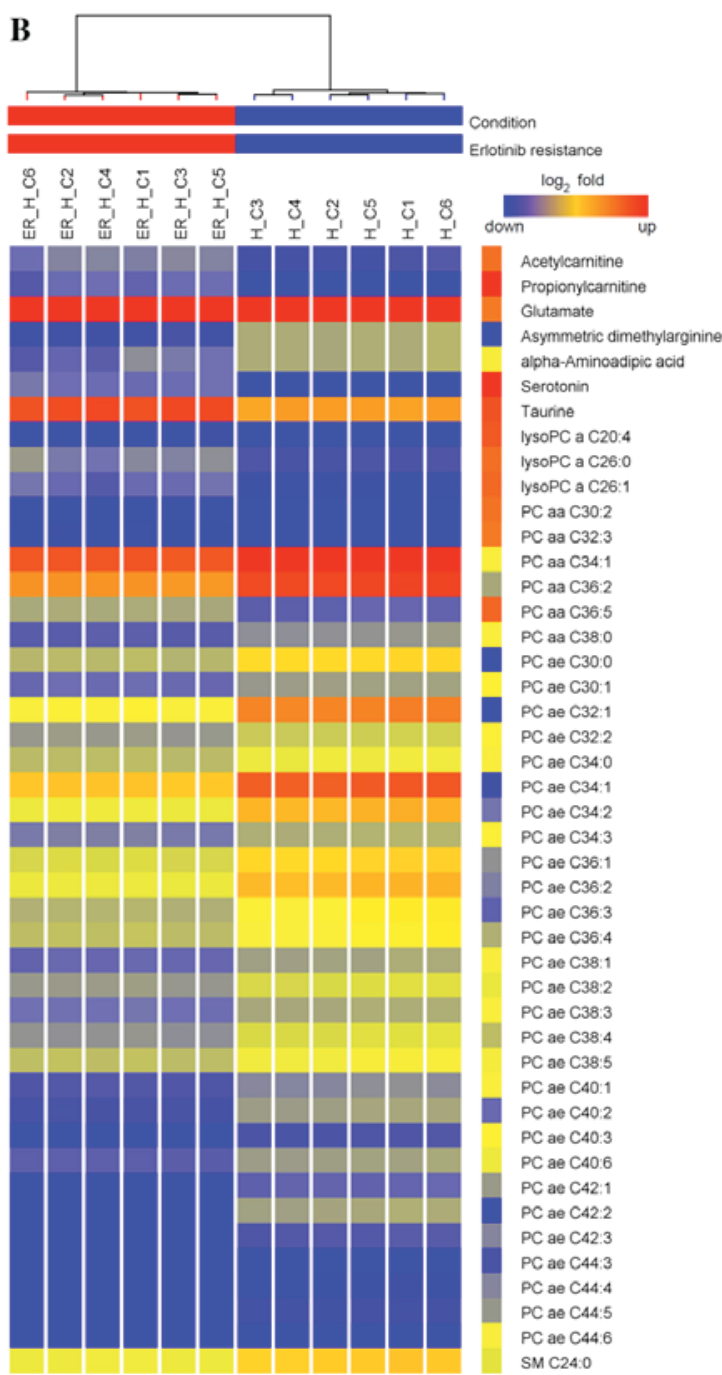

Figure 3. (A) Volcano plot for significantly differentially produced metabolites between HPAC and HPAC-ER cells. Blue triangles indicate that the metabolite was significantly decreased in terms of the P-value and $\log _{2}$ fold-change value; gray circles indicate that the metabolite level was not significantly altered in

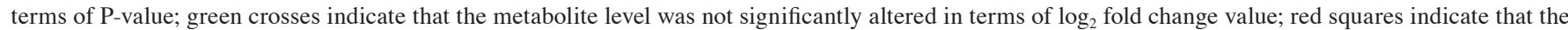
metabolite was significantly increased in terms of the P-value and $\log _{2}$ fold-change value. (B) Heat map of significantly differentially produced metabolites between HPAC and HPAC-ER cells, depicting the levels of metabolites in each sample and the variation in each metabolite between samples, ranging from blue (low intensity or downregulation) through to yellow and red (high intensity or upregulation. HPAC, human pancreatic adenocarcinoma cells; HPAC-ER, erlotinib-resistant HPAC cells. 
The present study revealed that the concentration of asymmetric dimethylarginine (ADMA) was elevated in HPAC-ER cells, whereas neither ADMA nor symmetric dimethylarginine (SDMA) were detected in the HPAC cells (Table II). Unlike SDMA, ADMA has been reported to be an endogenous inhibitor of nitric oxide synthase, which synthesizes nitric oxide from L-arginine (19). The ratio of arginine:ADMA:SDMA is used to monitor pathophysiological states in a variety of diseases, particularly cardiovascular diseases $(20,21)$. The level of arginine, the precursor of methylated arginine derivatives, including ADMA, was not significantly different between HPAC-ER and HPAC in the present study. Previous studies have reported a higher level of ADMA in blood samples from patients with colon cancer compared with those from healthy subjects $(22,23)$. Li et al (22) proposed that the increased level of ADMA in patients with colon cancer reflected the evasion of apoptosis exhibited by cancer cells in order to deal with cellular stressors, including chemotherapy.

The majority of metabolites quantified in the current study were lysophosphatidylcholines (lysoPCs) and PCs. The concentrations of 3/13 lysoPCs increased ( $\log _{2}$ fold-change range, 1.25-1.66), while those of 31/72 PCs decreased ( $\log _{2}$ fold-change range, -2.93 to -1.06$)$ in the HPAC-ER cells compared with the HPAC cells (Table II). Among 14 types of sphingomyeline (SM), SM C24:0 was significantly decreased in the HPAC-ER cells compared with the HPAC cells, whereas the levels of the other lipids were not significantly changed (Table II). Activation of phospholipase $\mathrm{A}_{2}$ serves an important role in carcinogenesis through enabling the hydrolysis of PCs to lysoPCs and arachidonic acid, which induce cancer cell growth and proliferation (24). Notably, the breakdown of PCs to lysoPCs and arachidonic acid has been observed in head and neck squamous cell carcinoma cells (25). Similarly, the current study observed elevated levels of lysoPCs and reduced levels of PCs in HPAC-ER cells compared with HPAC cells (Table II). It is well documented that choline phospholipid metabolism is markedly altered in the majority of cancer types $(25,26)$. These results suggested that the metabolic alterations observed in HPAC-ER cells in the present study, including elevated lysoPC and decreased PC levels, could be used as biomarkers for predicting resistance to erlotinib in pancreatic cancer.

The comparative metabolomic analysis of human pancreatic cancer cells, HPAC and HPAC-ER, in the present study revealed that metabolic alterations were associated with resistance to erlotinib human pancreatic cancer. Notably, significantly increased levels of short-chain acylcarnitines and lysoPCs, and significantly decreased levels of PCs, were identified in the HPAC-ER cells compared with the HAPC cells, indicating an increased phospholipid turnover. In addition, the observed metabolic changes support the theory that acetyl-CoA-associated and choline phospholipid metabolism serve important roles in pancreatic cancer development. Furthermore, the data from the present study revealed that glutamate, ADMA, $\alpha$-aminoadipic acid and taurine were significantly increased in the HPAC-ER cells, suggesting that there are higher metabolic demands in erlotinib-resistant cancer. Metabolic alterations in the specific amino acids discussed may aid in elucidating the biological mechanisms of erlotinib-resistance in pancreatic cancer.
In conclusion, the findings of the present study will contribute to the better understanding of the overall metabolic changes that occur in chemoresistant pancreatic cancer and will aid in associating particular metabolomes to specific cancer phenotypes, particularly those of chemoresistance. Despite the simultaneous determination of multiple groups of metabolites, the data from the present study is not sufficient to illustrate comprehensive metabolite changes. Nevertheless, the current study highlights the fact that the identification and characterization of metabolic markers may allow for the earlier detection of chemoresistance. This will allow for more rapid drug regimen changes, prolonging patient survival, particularly in pancreatic cancer.

\section{Acknowledgements}

The present study was supported by the Bio \& Medical Technology Development Program of the National Research Foundation (NRF) funded by the Ministry of Science, ICT \& Future Planning (grant no. NRF-2015M3A9E1028327) and the Basic Science Research Program through the National Research Foundation of Korea, which is funded by the Ministry of Education (grant nos. NRF-2014R1A1A1036222 and 2014R1A1A2054979).

\section{References}

1. Vincent A, Herman J, Schulick R, Hruban RH and Goggins M: Pancreatic cancer. Lancet 378: 607-620, 2011.

2. Hidalgo M: Pancreatic cancer. N Engl J Med 362: 1605-1617, 2010.

3. Moore MJ, Goldstein D, Hamm J, Figer A, Hecht JR, Gallinger S, Au HJ, Murawa P, Walde D, Wolff RA, et al: Erlotinib plus gemcitabine compared with gemcitabine alone in patients with advanced pancreatic cancer: A phase III trial of the National Cancer Institute of Canada Clinical Trials Group. J Clin Oncol 25: 1960-1966, 2007.

4. Bouhifd M, Hartung T, Hogberg HT, Kleensang A and Zhao L: Review: Toxicometabolomics. J Appl Toxicol 33: 1365-1383, 2013.

5. Cuperlović-Culf M, Barnett DA, Culf AS and Chute I: Cell culture metabolomics: Applications and future directions. Drug discovery today 15: 610-621, 2010 .

6. Theodoridis GA, Gika HG, Want EJ and Wilson ID: Liquid chromatography-mass spectrometry based global metabolite profiling: A review. Anal Chim Acta 711: 7-16, 2012.

7. Freedman VH and Shin SI: Cellular tumorigenicity in nude mice: Correlation with cell growth in semi-solid medium. Cell 3: 355-359, 1974.

8. Matuszewski BK, Constanzer ML and Chavez-Eng CM: Strategies for the assessment of matrix effect in quantitative bioanalytical methods based on HPLC-MS/MS. Anal Chem 75: 3019-3030, 2003.

9. Kerner J and Hoppel C: Fatty acid import into mitochondria. Biochim Biophys Acta 1486: 1-17, 2000.

10. Zammit VA, Ramsay RR, Bonomini M and Arduini A: Carnitine, mitochondrial function and therapy. Adv Drug Deliv Rev 61: 1353-1362, 2009.

11. Medina MA, Sánchez-Jiménez F, Márquez J, Rodríguez Quesada A and Núñez de Castro I: Relevance of glutamine metabolism to tumor cell growth. Mol Cell Biochem 113: 1-15, 1992.

12. Sasada S, Miyata Y, Tsutani Y, Tsuyama N, Masujima T, Hihara J and Okada M: Metabolomic analysis of dynamic response and drug resistance of gastric cancer cells to 5-fluorouracil. Oncol Rep 29: 925-931, 2013.

13. Staubert C, Bhuiyan H, Lindahl A, Broom OJ, Zhu Y, Islam S, Linnarsson S, Lehtiö J and Nordström A: Rewired metabolism in drug-resistant leukemia cells: A metabolic switch hallmarked by reduced dependence on exogenous glutamine. J Biol Chem 290: 8348-8359, 2015 . 
14. Serizawa M, Kusuhara M, Zangiacomi V, Urakami K, Watanabe M, Takahashi T, Yamaguchi K, Yamamoto N and Koh Y: Identification of metabolic signatures associated with erlotinib resistance of non-small cell lung cancer cells. Anticancer Res 34: 2779-2787, 2014.

15. Fujimura Y, Ikenaga N, Ohuchida K, Setoyama D, Irie M, Miura D, Wariishi H, Murata M, Mizumoto K, Hashizume M and Tanaka M: Mass spectrometry-based metabolic profiling of gemcitabine-sensitive and gemcitabine-resistant pancreatic cancer cells. Pancreas 43: 311-318, 2014.

16. Ripps H and Shen W: Review: Taurine: A 'very essential' amino acid. Mol vis 18: 2673-2686, 2012.

17. Bellance N, Pabst L, Allen G, Rossignol R and Nagrath D: Oncosecretomics coupled to bioenergetics identifies $\alpha$-amino adipic acid, isoleucine and GABA as potential biomarkers of cancer: Differential expression of c-Myc, Oct1 and KLF4 coordinates metabolic changes. Biochim Biophys Acta 1817: 2060-2071, 2012.

18. Peters MA, Walenkamp AM, Kema IP, Meijer C, de Vries EG and Oosting SF: Dopamine and serotonin regulate tumor behavior by affecting angiogenesis. Drug Resist Updat 17: 96-104, 2014.

19. Beltowski J and Kedra A: Asymmetric dimethylarginine (ADMA) as a target for pharmacotherapy. Pharmacol Rep 58: 159-178, 2006

20. Davids M, Swieringa E, Palm F, Smith DE, Smulders YM, Scheffer PG, Blom HJ and Teerlink T: Simultaneous determination of asymmetric and symmetric dimethylarginine, L-monomethylarginine, L-arginine and L-homoarginine in biological samples using stable isotope dilution liquid chromatography tandem mass spectrometry. J Chromatogr B Analyt Technol Biomed Life Sci 900: 38-47, 2012.
21. Martens-Lobenhoffer J and Bode-Böger SM: Quantification of L-arginine, asymmetric dimethylarginine and symmetric dimethylarginine in human plasma: A step improvement in precision by stable isotope dilution mass spectrometry. J Chromatogr B Analyt Technol Biomed Life Sci 904: 140-143, 2012.

22. Li H, Zhou Y, Zhao A, Qiu Y, Xie G, Jiang Q, Zheng X, Zhong W, Sun X, Zhou Z and Jia W: Asymmetric dimethylarginine attenuates serum starvation-induced apoptosis via suppression of the Fas (APO-1/CD95)/JNK (SAPK) pathway. Cell Death Dis 4: e830, 2013

23. Yoshimatsu M, Toyokawa G, Hayami S, Unoki M, Tsunoda T, Field HI, Kelly JD, Neal DE, Maehara Y, Ponder BA, et al: Dysregulation of PRMT1 and PRMT6, Type I arginine methyltransferases, is involved in various types of human cancers. Int J Cancer 128: 562-573, 2011.

24. Cummings BS: Phospholipase A2 as targets for anti-cancer drugs. Biochem Pharmacol 74: 949-959, 2007.

25. Tripathi P, Kamarajan P, Somashekar BS, MacKinnon N, Chinnaiyan AM, Kapila YL, Rajendiran TM and Ramamoorthy A: Delineating metabolic signatures of head and neck squamous cell carcinoma: Phospholipase A2, a potential therapeutic target. Int J Biochem Cell Biol 44: 1852-1861, 2012.

26. Iorio E, Mezzanzanica D, Alberti P, Spadaro F, Ramoni C, D'Ascenzo S, Millimaggi D, Pavan A, Dolo V, Canevari S and Podo F: Alterations of choline phospholipid metabolism in ovarian tumor progression. Cancer Res 65: 9369-9376, 2005. 